\title{
Where Should Radiotherapy Stand in the Current era of Rectal Cancer Management?
}

- Duygu SEZEN, ${ }^{\oplus} \odot$ Nülifer KILIÇ DURANKUŞ, ${ }^{1} \odot$ Yasemin BÖLÜKBAŞI,1,2,3 $\odot$ Uğur SELEK 1,2,3

Department of Radiation Oncology, Koç University, School of Medicine, Istanbul-Turkey

Department of Radiation Oncology, American Hospital, UT MD Anderson Radiation Treatment Center, Istanbul-Turkey

Department of Radiation Oncology, HospitalUniversity of Texas M.D. Anderson Cancer Center, Houston,TX-ABD

\section{SUMMARY}

Rectal cancer management can be defined as maximizing local tumor control and overall survival while preserving anal sphincter, bladder, and sexual functions or improving the quality of life with an overall reduction in morbidity. Appropriate preoperative or postoperative therapy is required to minimize the risks of both local and distant recurrence. Preoperative radiotherapy is the current standard for treating patients with high-risk rectal cancer owing to lower rates of local relapse and toxicity. Modern radiotherapy capabilities are well suited for any short- or long-course protocol with decreased toxicity in irradiated structures such as the small intestine, bladder, or femoral heads. As clinicians and researchers, we must aim to establish tailored treatments for these patients based on the most suitable evidence based ground in a multidisciplinary environment regarding the expectations of both our patients and team physicians. Herein, we present a review of ongoing clinical trials in order to shed light on the current debates of standard approaches for treating rectal cancer.

Keywords: Long course; neoadjuvant; non-operative management; preoperative; rectal cancer; short course; tota neoadjuvant.

Copyright $\odot$ 2018, Turkish Society for Radiation Oncology

\section{Introduction}

Rectal cancer management can be simply defined as maximizing local tumor control and overall survival while preserving anal sphincter, bladder, and sexual functions or improving the quality of life with an overall reduction in morbidity.

It is well known that different risks of both local and distant recurrence mandate a tailored approach, including appropriate preoperative or postoperative herapy; therefore, all subsequent modalities and their timings must be optimized according to prognostic evaluation. The prognosis of patients with rectal cancer is determined mostly based on defined factors. The most important prognostic criteria are provided by histopathology. TNM staging is independent prognostic factor according to multivariate analysis, whereas size differentiation, and vascular invasion are independen prognostic factors according to univariate analysis. The following parameters should be assessed when initially deciding the local recurrence risk: $\mathrm{T}$ stage with depth of extramural spread in $\mathrm{mm}, \mathrm{N}$ stage with ymph node involvement load, extramural vascul invasion, circumferential resection margin status, and peritoneal perforation caused by the tumor. Tumo stage T3 increases local recurrence risk, thereby resulting in prognostic inhomogeneity. Patients with T tumors have been demonstrated to have a significantly

\section{Received: May 23, 2018

onger cancer-specific survival if extramural invasion is less than $5 \mathrm{~mm}$ in pathology (5-year survival rate: $85 \%$ vs. $54 \%$ ).[1] The risk propagates with increasing tumor invasion into the perirectal fat to increase nodal involvement.[2] Lymph node involvement has long been identified as an independent adverse prognostic factor.[3] The extent of nodal involvement with four or more tumor-positive nodes has started to be more relevant for pelvic recurrence after total mesorectal excision (TME) in comparison to any nodal involven predicting recurrence before TME.[4]

The involvement of circumferential resection margin (CRM) is also an independent prognostic factor,2 and its significance for local recurrence, distant metastases, and survival persists despite TME. $[5,6]$ TME is the current standard of surgery as the middle and lower rectum are resected together with the mesorectum,[7-9] and this surgical approach is said to decrease local recurrence, [7-12] along with a decrease in positive CRM Therefore, patients with potentil for CRM positity should be given with potential for CRM posivity should be given neadjuvant treatment to decrease related risks. The incidence of positive circumferential radial margins in a Polish study was found to be lower after long-course chemoradiotherapy in comparison to short-course radiotherapy (4\% vs $13 \%, \mathrm{P}=0.017$ ).[13] If the final pathology defines an involved CRM, postoperative treatment unfortunately appears to have a limited ability to compensate, as reported in a subset analysis of the Dutch CKVO trial, which demonstrated the inefficacy of ostoperative long-course radiation alone to decrease local recurrences,[14] and in the MRC CR-07 trial, which revealed a local recurrence rate of $11 \%$ despite postoperative long-course chemoradiotherapy.[15]

Evolution of Preoperative Radiotherap

We have shed light on the discussion of preoperative versus postoperative radiotherapy first with two phase 3 trials defining decreasing local recurrence and complication rates with preoperative radiotherapy [16,17] pence $[16,17]$ A Swedish trial $5 \times 5$ Gy preoperative radiotherapy ane versus 60 Gy postoperative radiotherapy randomizing 471 patients reported a significant decrease in local recurrence (5-year recurrence: preop 13\%, postop 22\%) and complication rates related with obstruction (5-year: preop 5\%, postop 11\%).[16] These results were confirmed 13 years later with a German chemoradiotherapy trial of preoperative (50.4 Gy and PVI $1 \mathrm{mg} / \mathrm{m}^{2} /$ day $5 \mathrm{FU}$ 1st, 5th weeks) versus postoperative ( $50.4 \mathrm{GY}+5.4$ GY boost and PVI $1 \mathrm{mg} / \mathrm{m}^{2} / \mathrm{day}$ $5 \mathrm{FU} 1$ st, 5th weeks) radiotherapy randomizing $823 \mathrm{pa}-$ tients.[17] This trial delineated the superiority of the preoperative approach with a decrease in local recurrence (5-year recurrence: preop 6\%, postop 13\%) and acute and late complication rates (acute: preop 27\% postop $40 \%$ : late: preop $14 \%$, postop $24 \%$ ). The overall survival did not differ in both trials.

The utility of radiotherapy in rectal cancers was questioned via two important meta-analyses, and its robust role has been verified. [18,19] The Colorectal Cancer Collaborative Group evaluated 22 trials, including 8500 cases, and concluded that both preoperative (46\% decrement in local recurrence) and postoperative (37\% decrement in local recurrence) radiotherapy provides local control benefit over surgery alone.[18] The Swedish Council of Technology Assessment in Health Care reported their analysis of 42 randomized studies, 3 meta-analyses, and 36 prospective and 7 retrospective studies (including 25000 cases) and concluded that preoperative radiotherapy ensures better local control in comparison to postoperave blo loc [19] It should be noted thastoere that preoperative single-modality radiotherapy significantly increased overall survival by $10 \%$, whereas postoperative radiotherapy failed to reach significance withou chemotherapy.

Sphincter preservation was also an issue to be questioned in preoperative chemoradiotherapy trials. The two randomized trials of conventionally fractionated preoperative versus postoperative chemoradiotherapy for clinically resectable rectal cancer reported contradictory results for sphinct preservation: sphincte preservation significantly increased in the German trial ( $39 \%$ vs $20 \%, \mathrm{P}=0.004) 17$; no significance wa reported in the NSABP R-03 trial ( $48 \%$ vs 39\%).[20] As the NSABP trial had limitations in statistical power due to low accrual (267 patients of the 900 planned) the German trial delineates the standard for sphincter preservation using preoperative chemoradiotherapy. Although $18 \%$ of patients clinically staged as T3No in the German trial who underwent initial surgery without neodjuvant treatment were found to be $1-2 \mathrm{NO}$ with overtreatment debates pe revealed that $22 \%$ of clinically staged T3N0 patients who completed neoadjuvant chemoradiotherapy were proved to be ypN+.[21]

Short Course versus Long Cours

Preoperative radiotherapy has been shown to be preferable to postoperative radiotherapy with lower rate of local relapse and toxicity $[16,17]$ The regimens dif- 
fer in the preoperative radiotherapy approach, whereas short course is preferred in Northern Europe and long course in Southern Europe and America. Neoadjuvant radiotherapy has been developed to offer two regimens that could be accepted as standards for resectable rectal cancer: short course 25 Gy (5×5 Gy) radiation therapy alone and long-course chemoradiation therapy.

Three major studies have shaped the literature on the use of preoperative radiotherapy alone: Rotterdam-Holland, EORTC, and Swedish trials.[22-24] The Rotterdam and EORTC trials evaluated $34.5 \mathrm{~Gy}$ (2.3Gy/fraction/day) preoperative radiotherapy and revealed local control benefit besides subgroup overall survival benefit for patients with T3-4 tumors resected curatively.[22,23] The Swedish trial was the first randomized study demonstrating overall survival benefit for all cohorts with preoperative radiotherapy. [24] A total of 1168 patients with clinically resectable rectal cancer were randomized to $25 \mathrm{~Gy}(5 \mathrm{~Gy} / \mathrm{frac}-$ tion/day) preoperative radiotherapy and immediate surgery in 1 week versus surgery alone; local tumor control and overall survival benefit was obtained with preoperative radiotherapy. In the TME era, a Dutch trial evaluated the same protocol and indicated a significant decrease in local failures (surgery alone: $8.2 \%$ vs. radiotherapy+surgery: $2.4 \%$ ) and noted a longer follow-up requirement for survival.[25]

Randomized trials testing short-course neoadjuvant radiotherapy accumulated evidence to be safe and efficient (Stockholm 1 [26], Stockholm 2 [27], Swedish trial [28], $5 \times 5$ Gy with immediate surgery vs surgery alone; Uppsala, $5 \times 5.1$ Gy with immediate surgery vs postoperative radiotherapy, $60 \mathrm{~Gy}, 2 \mathrm{~Gy}$ per fraction [16]; Dutch TME trial [14,29], MRC CR07 [15], 5×5 Gy with immediate surgery vs surgery alone or postoperative radiotherapy for high-risk patients; Stockholm III trial [30], 5×5 Gy with immediate surgery as group 1 vs $5 \times 5$ Gy with surgery after $4-8$ weeks as group 2 vs $25 \times 2$ Gy with surgery after $4-8$ weeks as group 3 ). The Stockholm III trial recently concluded that shortcourse radiotherapy with delay in surgery appeared to be a convenient alternative to conventional short-course radiotherapy with immediate surgery.[31]

Swedish trials [26-28] comparing surgery alone with neoadjuvant short-course radiotherapy and immediate surgery identified that radiotherapy reduced local recurrence ranging from $52 \%$ to $65 \%$ as well as resulted in an absolute overall survival benefit of $8 \%$ at 13 years. [28] Even in the TME era, randomized studies of the Dutch TME trial $[14,29]$ and Medical
Research Council (MRC) CR07 trial [15] showed an approximately $50 \%-60 \%$ relative reduction of local recurrence after short-course preoperative radiotherapy with an absolute local control benefit of 5\%-6\%, whereas no overall survival benefit with radiation was found this time.

The literature was lacking randomized trials comparing neoadjuvant short-course radiotherapy with neoadjuvant chemoradiotherapy for T3 cancers until the results of two trials questioning this dileme: [13 32-35] a Polisho tilemma: (uifferences in the rates of sphincter-preserving surgery between long-course chemoradiation and short-course radiotherapy, and an Australian study, which evaluated differences in local recurrence rates between these arms. Both trials demonstrated significantly increased early radiation toxicity in the chemoradiation group (grade 3-4 acute toxicity rates, Polish: $18 \%$ vs. $3 \%$; Australian: $28 \%$ vs. $1.9 \%$ ), which turned into improved adherence to the protocol in short-course radiation only arms. Intere Interestingy the sphere similar in both arms of the Polish trial (short: $61 \%$ and long: 58\%), whereas the local recurrence rate was lower in the short arm (short: $10.6 \%$ and long: $15.6 \%$ ). Although the follow-up is yet limited, no significan differences were observed between the randomized groups regarding survival, postoperative complications, late toxicity rates (severe late toxicity, Polish $10.1 \%$ vs. $7.1 \%$; Australian: $7.6 \%$ vs. $8.8 \%$ ), quality of life, and anorectal and sexual functions.

There has been a debate about whether preoperative chemoradiotherapy or short-course radiotherapy is preferable for patients with threatened CRMs and those with low-lying cancer to increase the chance of anterior resection. $[14,36]$ There is a consensus th neoadjuvant chemoradiotherapy is the treatment of choice for unresectable cancers, but the major conflict is for resectable lesions with threatened CRMs. The recent two trials did not exclude patients wit resectable tumors having involved CRMs, and the resectable thors have Dutch TME (18.2\% of enrolled patients) [14] and MRC CR07 trials (10.8\% of enrolle patients) [15] revealed that the local recurrence rates were lower in neoadjuvant short-course radiotherapy arms in comparison to selective postoperative chemoradiotherapy arms (Dutch: $9.3 \%$ vs $16.4 \%$ and MRC $13.8 \%$ vs $20.7 \%$ ). Based on this data, short-course preoperative radiotherapy appeared to be more effective than selective conventionally fractionated postoperative radiotherapy or chemoradiotherapy in case of resectable cancer with threatened CRMs in preoperative imaging. Besides, the Polish study [13] as well as the two systematic reviews $[37,38]$ could not discover any improvement with neoadjuvant chemoradiotherapy in terms of sphincter preservation in comparison to neoadjuvant short-course radiotherapy alone despite the hypothesis of better reduction in tumor bulk after chemoradiotherapy for anterior resection to spare sphincter function.

Overall, the combined MRC CR07/NCIC-CTG C016 trial randomized 1350 patients with rectal cancer to preoperative short-course radiation of 25 Gy $(5 \times 5$ Gy) or selective postoperative chemoradiation of 45 $\mathrm{Gy}(25 \times 1.8 \mathrm{~Gy})$ with concurrent $5 \mathrm{FU}$ for patients with an involved CRM. [15,39] Although no overall survival benefit was outlined between the two arms, this study noted $61 \%$ reduction in the relative risk of local recurrence with preoperative radiotherapy besides an absolute difference of $6.2 \%$ at 3 years (local recurrence with preoperative radiotherapy: $4.4 \%$; postoperative chemoradiotherapy: 10.6\%) and 24\% improvement in isease-free survival with preope disease-free sreoperative radiotherapy vesides an absolute difference of $6 \%$ at 3 years $(77.5 \%$ vs $71.5 \%) .[15,39]$ The importance of achieving a negative CRM was also confirmed with special notification to the plane of surgery as an independent predictor of local recurrence according to multivariate analysis, and involved margins caused a three-fold increased risk of local recurrence ( $17 \%$ vs. $6 \%)$ and a reduced 3 -year disease-free survival ( $50 \%$ vs. $79 \%$ ). [15,39] This study delineated important aspects that affect the adjuvant treatment appr uvant teatment appoach: local recurence rates are lower in upper-third rectal cancers in comparison to lower-third tumors, and recurrence rates increase with more advanced TNM stage as well as with involvement of CRM. The CR07/C016 trial declared that surgery should be performed correctly, adjuvant radiation should be considered preoperatively, and short-term radiotherapy alone is safe and efficient.

The recent phase III Polish II trial for cT4 or fixed CT3 rectal cancer investigated the comparison of longcourse preoperative chemoradiation of 50.4 Gy in 28 fractions combined with two 5 -day cycles of bolus 5 -FU $325 \mathrm{mg} / \mathrm{m}^{2} /$ day and leucovorin $20 \mathrm{mg} / \mathrm{m}^{2} / \mathrm{day}$ during the first and fifth week of irradiation along with five infusions of oxaliplatin $50 \mathrm{mg} / \mathrm{m}^{2}$ once weekly versus short-course $5 \times 5$ Gy and three cycles of consolidation FOLFOX4 chemotherapy.[40] Bujko et al. revealed no differences in local efficacy between both arms but reported an improved overall survival and solidation chemotherapy.[40]
The RAPIDO phase 3 trial is open to accrual for locally advanced rectal cancer randomizing the standard arm of chemoradiation (1.8 Gy $\times 25$ or $2 \mathrm{~Gy} \times 25$ with capecitabine) preoperatively followed by selective postoperative adjuvant chemotherapy of eight cycles of CAPOX versus short-course radiotherapy followed by six cycles of neo-adjuvant CAPOX chemotherapy.[41]

\section{Time to Surgery}

Interval to surgery after preoperative radiotherapy/ chemoradiotherapy is a direct correlation with tumor down-staging and interval to surgery after radiotherapy,[42-44] and therefore, long-course chemoradiotherapy has long been generally preferred in initially unresectable tumors to gain downsizing. [13,45-48] Prospective studies evaluating the rates of sphincter preservation by neoadjuvant chemoradiotherapy determined a change in the surgical approach from abdominoperineal resection to sphincter preservation in 23\%-85\% (median: 75\%), whereas the complete pathrecthlocal recurrence rates were $0 \%-17 \%$. [4-54] The interval to surgery for postoperative radiotherapy was 2 weeks versus 6-8 weeks in a Lyon study and the complete surgical response was $10 \%$ at 2 weeks and $26 \%$ at $6-8$ weeks. [42] However, the pathological response increased when surgery was delayed even for short-course $5 \times 5$ Gy regimen in unresectable cancers.[30,55-56] The appropriate interval after chemoradiation for surgery timing was assessed in multiple retrospective database studies a stice (NCDB) and weeks were associated with increased complete pathological response rates without any increase in surgical complications, [57] higher odds of positive margins and plateauing pathological down-staging, [58] in creased odds of positive surgical margins in addition to decreased rate of sphincter preservation, and increased risk of death.[59] GRECCAR-6 is a multicenter, ran domized, controlled trial conducted for patients with rectal cancer to evaluate the effect of interval between

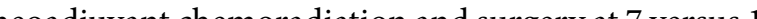
weeks on complete pathogical response the resuls of the trial reveled no difference in the comple pasological response week group (6) TME and more

Selective Use of Radiotherapy

The general hypothesis was to avoid radiotherapy after an objective good clinical response in a subset of pa- 
tients receiving neoadjuvant chemotherapy. The initial effort was made in a pilot phase 2 MSKCC study enrolling 32 patients [61] who were candidates for low anterior resection with TME and received six cycles of FOLFOX, with bevacizumab included for cycles 1 to 4 and were planned to undergo radiation before TME if stable/progressive disease detected, whereas responders were to undergo immediate TME; postoperative radiation was administered if $\mathrm{R} 0$ resection could not be achieved. All study participants underwent R0 resections, where whereas two were withdrawn due to cardiac events during chemotherapy and received preoperative chemoradiotherapy. The 4-year disease-free survival was $84 \%$, which did not seem to compromise outcomes and encouraged neoadjuvant chemotherapy and selective radiation for selected patients with clinically staged II-III rectal cancer. Therefore, a randomized phase III trial named "Preoperative Radiation or Selective Preoperative Radiation and Evaluation Before Chemotherapy and TME (PROSPECT)" is open to vilate this experience by (PROSPECI)" is open to validate this experience by conparing 5 -fluorouracil or capecitabine and 5.5 weeks of radiation therapy followed by TME and adjuvant therapy of eight cycles of FOLFOX versus completing FOLFOX chemotherapy once every 2 weeks for six cycles over a total of 12 weeks and MRI or endorectal ultrasound response by $20 \%$ or more proceeding to TME or by less than $20 \%$ receiving $5 \mathrm{FU}$ chemoradiotherapy then TME completed with adjuvant FOLFOX after surgery.

\section{Total Neoadjuvant Therap}

Neoadjuvant therapy has been also investigated as a total preoperative therapy with upfront rather than adjuvant chemotherapy to further improve outcomes by addressing possible micrometastatic disease as well as the primary tumor. Two phase II studies, UK and Spain trials, have evaluated induction chemotherapy followed by preoperative CRT in high-risk patients based on MRI for assessing the extent of extramural tumor invasion and risk of CRM positivity [62,63] Induction CAPOX chemotherapy befo CAPOX Che and Spanish GCR-3 thials had similar pr plete resection rates in comparison to postoperative adjuvant CAPOX, whereas more favorable compliance and toxicity profiles were achieved $[63,64]$ Similarly, in the MSKCC study, total neoadjuvant radiotherapy with FOLFOX and chemoradiation followed by planned TME resulted in a considerable rate of pathCR and delivery of planned therapy in addition to offering a very selective decent stand for possible non-operative management.[65] mFOLFOX6 chemotherapy after concur- rent chemoradiation before TME has also shown to potentially increase the pCR up to $38 \%$.[66]

A recently proposed NRG-GI002 phase II clinical trial platform will be randomizing phase II modular clinical trials utilizing total neoadjuvant therap with parallel experimental arms.[67] Besides Trial Evaluating 3-year Disease-Free Survival in Patient With Locally Advanced Rectal Cancer Treated With Chemoradiation Plus Induction or Consolidation and Total Mesorectal Excision or Nonoperative Management

\section{Non-operative Management (NOM)}

Non-operative management (NOM) has been trending as a paradigm shift to avoid surgery if possible in case of complete clinical response to preoperative treatment, following neoadjuvant Brazilian CRT data which define safe and good survival rates in a highly selected group of patients without surgery because 99 f 122 ptients with complete clinical response (CR at first response assessment sustained cCR for a minimum of 12 months were managed nonoperatively with stage $\mathrm{c} 0$ and at a mean follow-up of 59.9 months, $13.1 \%$ recurred (5\% endorectal, $7.1 \%$ systemic, $1 \%$ combined).[68] An update of this data by Habr-Gam in 2013 continued to encourage NOM in selected patients. [69,70] Dutch data reinforced the NOM approach with strict selection criteria and frequent follow-up of endoscopy and MRI for organ preservatio as oncologically safe for selected $C \mathrm{CR}$ or near $\mathrm{CCR}$ after [71,72] The 3-yer afterneoadjuvant chemoradiation. $[71,72]$ The 3-year overall survival rates, distant metastasis-free survival rate local regrowth-free survival rates, and disease-fre survival rates were $96.6 \%, 96.8 \%, 84.6 \%$, and $80.6 \%$, respectively.[72]

Because surgery is yet the only reliable method to detect a pCR and $\mathrm{CCR}$ does not mean pathologic response, an intensive effort to distinguish post-RT changes from residual disease, continuous evaluation of digital rectal examination, endoscopic assessment chI int post treatment biopsies must be prospectively investigated until NOM in rectal cancer can b considered a standard approach. NCT02008656 (Tria Evaluating 3-year Disease-Free Survival in Patient With Locally Advanced Rectal Cancer Treated With Chemoradiation Plus Induction or Consolidation Chemotherapy and Total Mesorectal Excision or Nonoperative Management) [73] and NCT02052921 (Observation Versus Surgical Resection in Patients With Rectal Cancer Who Achieved Complete Clinical Re- sponse After Neoadjuvant Chemoradiotherapy) [74] are ongoing for accrual.

\section{Summary}

It is well known that different risks of both local and distant recurrence mandate an individualized approach, including appropriate preoperative or postoperative therapy. Preoperative radiotherapy is the current standard for treating patients with high-risk rectal cent stance cancer because icity. Modern radiotherapy capabilities are well suited
for any short- or long-course protocol with decreased for any short- or long-course protocol with decreased
toxicity in irradiated structures such as the small intoxicity in irradiated structures such as the small in-
testine, bladder, or femoral heads. As clinicians and retestine, bladder, or femoral heads. As clinicians and researchers, we must aim to establish tailored treatments for these patients based on the most suitable evidence garding the expectations of both our patients and team physicins. In this review, we have shed light on the current debates of a standard treatment approach for rectal cancer in ongoing clinical trials.

Peer-review: Externally peer-reviewed.

Conflict of Interest: None declared.

Authorship contributions: Concept - U.S.; Design - D.S. Supervision - Y.B.; Data collection \&/or processing - N.K.D. Analysis and/or interpretation - U.S.; Literature search N.K.D.; Writing - D.S.; Critical review - Y.B.

\section{References}

1. Merkel S, Mansmann U, Siassi M, Papadopoulos T, Hohenberger W, Hermanek P. The prognostic inhomogeneity in $\mathrm{pT} 3$ rectal carcinomas. Int I Colorectal Dis 2001;16(5):298-304.

2. Quirke P, Durdey P, Dixon MF, Williams NS. Local recurrence of rectal adenocarcinoma due to inadequate surgical resection. Histopathological study of equate surgical resection. Histopathological study of 1986;2(8514):996-9.

3. Dukes CE. The classification of cancer of the rectum. J Pathol Bacteriol 1932;35:323-32

4. Hermanek P, Merkel S, Fietkau R, Rödel C, Hohenberger W. Regional lymph node metastasis and locoregional recurrence of rectal carcinoma in the era of TME [corrected] surgery. Implications for treatment decisions. Int J Colorectal Dis 2010;25(3):359-68.

5. Wibe A, Rendedal PR, Svensson E, Norstein J, Eide TJ, Myrvold HE, et al. Prognostic significance of the circumferential resection margin following to- tal mesorectal excision for rectal cancer. Br J Surg 2002;89(3):327-34

6. Hall NR, Finan PJ, al-Jaberi T, Tsang CS, Brown SR Dixon MF, et al. Circumferential margin involvement after mesorectal excision of rectal cancer with curative intent. Predictor of survival but not local recurrence? Dis Colon Rectum 1998;41(8):979-83.

7. Heald RJ, Husband EM, Ryall RD. The mesorectum in rectal cancer surgery-the clue to pelvic recurrence? $\mathrm{Br}$ J Surg 1982;69(10):613-6.

8. Heald RJ. Total mesorectal excision. The new European gold standard. G Chir 1998;19(6-7):253-5.

9. Heald RJ. Surgical management of rectal cancer: a multidisciplinary approach to technical and technological advances. Br J Radiol 2005;78 Spec No 2:S128-30.

10. Leong AF. Selective total mesorectal excision for rectal cancer. Dis Colon Rectum 2000;43(9):1237-40

11. Merchant NB, Guillem JG, Paty PB, Enker WE, Min sky BD, Quan SH, et al. T3N0 rectal cancer: results following sharp mesorectal excision and no adjuvan therapy. J Gastrointest Surg 1999;3(6):642-7.

12. MacFarlane JK, Ryall RD, Heald RJ. Mesorectal excision for rectal cancer. Lancet 1993;341(8843):457-60.

13. Bujko K, Nowacki MP, Nasierowska-Guttmejer A Michalski W, Bebenek M, Pudełko M, et al. Sphincter preservation following preoperative radiotherapy for rectal cancer: report of a randomised trial comparing short-term radiotherapy vs. conventionally fractionated radiochemotherapy. Radiother Once 2004;72(1):15-24.

14. Marijnen CA, Nagtegaal ID, Kapiteijn E, Kranenbarg EK, Noordijk EM, van Krieken JH, et al. Radiotherapy does not compensate for positive resection margins in rectal cancer patients: report of a multicenter randomized trial. Int J Radiat Oncol Biol Phys 2003;55(5):1311-20.

15. Sebag-Montefiore D, Stephens RJ, Steele R, Monson J, Grieve R, Khanna S, et al. Preoperative radiotherapy versus selective postoperative chemoradiotherapy in patients with rectal cancer (MRC CR07 and NCICCTG C016): a multicentre, randomised trial. Lancet 2009:373(9666):811-20.

16. Frykholm GJ, Glimelius B, Påhlman L. Preoperative or postoperative irradiation in adenocarcinoma of the rectum: final treatment results of a randomized trial and an evaluation of late secondary effects. Dis Colon Rectum 1993;36(6):564-72.

17. Sauer R, Becker H, Hohenberger W, Rödel C, Wittekind $\mathrm{C}$, Fietkau R, et al. Preoperative versus postoperative chemoradiotherapy for rectal cancer. $\mathrm{N} \mathrm{Engl}$ Med 2004;351(17):1731-40.

18. Colorectal Cancer Collaborative Group. Adjuvan 
radiotherapy for rectal cancer: a systematic overview of 8,507 patients from 22 randomised trials. Lancet 2001;358(9290):1291-304.

19. Glimelius B, Grönberg H, Järhult J, Wallgren A, Cavallin-Ståhl E. A systematic overview of radiation therapy effects in rectal cancer. Acta Oncol 2003;42(5-6):47692.

20. Roh MS, Colangelo LH, O'Connell MJ, Yothers G, Deutsch M, Allegra CJ, et al. Preoperative multimodality therapy improves disease-free survival in patients with carcinoma of the rect

21.Guillem JG, Díaz-González JA, Minsky BD, Valentini V, Jeong SY, Rodriguez-Bigas MA, et al. cT3N0 tini $V$, Jeong SY, Rodriguez-Bigas MA, et al. cT3NO
rectal cancer: potential overtreatment with preoprectal cancer: potential overtreatment with preop2008;26(3):368-73.

22. Wassif SB. The role of pre-operative adjuvant therapy in the management of borderline operability rectal cancer. Clin Radiol 1982;33(3):353-8.

23. Gérard A, Buyse M, Nordlinger B, Loygue J, Pène F, Kempf P, et al. Preoperative radiotherapy as adjuvant treatment in rectal cancer. Final results of a randomized study of the European Organization for Revearch and Treatment of Cancer (EORTC). Ann Surg search and Treatment

24. Swedish Rectal Cancer Trial, Cedermark B, Dahlberg M, Glimelius B, Påhlman L, Rutqvist LE, Wilking N. Improved survival with preoperative radiotherapy in resectable rectal cancer. $\mathrm{N}$ Engl J Med 1997;336(14):980-7.

25. Kapiteijn E, Marijnen CA, Nagtegaal ID, Putter H, Steup WH, Wiggers T, et al. Preoperative radiotherapy combined with total mesorectal excision for resectable rectal cancer. N Engl J Med 2001;345(9):638-46.

26. Cedermark B, Johansson H, Rutqvist LE, Wilking N. The Stockholm I trial of preoperative short term radiotherapy in operable rectal carcinoma $A$ prospective therapy in op andomized trial. Stockholm Colorecta

27. Martling A, Holm T, Johansson H, Rutqvist LE, Cedermark B; Stockholm Colorectal Cancer Study Group The Stockholm II trial on preoperative radiotherapy in rectal carcinoma: long-term follow-up of a popultion-based study. Cancer 2001;92(4):896-902.

28. Folkesson J, Birgisson H, Pahlman L, Cedermark B, Glimelius B, Gunnarsson U. Swedish Rectal Cancer Trial: long lasting benefits from radiotherapy on survival and local recurrence rate. J Clin Oncol 2005;23(24):5644-50.

29. Peeters KC, Marijnen CA, Nagtegaal ID, Kranenbarg EK, Putter H, Wiggers T, et al. The TME trial after a median follow-up of 6 years: increased local contro but no survival benefit in irradiated patients with resectable rectal carcinoma. Ann Surg 2007;246(5):693 701.

30. Pettersson D, Cedermark B, Holm T, Radu C, Påhlman L, Glimelius B, et al. Interim analysis of the Stockholm III trial of preoperative radiotherapy regimens for rectal cancer. Br J Surg 2010;97(4):580-7.

31. Erlandsson J, Holm T, Pettersson D, Berglund A Cedermark B, Radu C, et al. Optimal fractionation of preoperative radiotherapy and timing to surgery for preoperative radiotherapy and timing to surgery for rectal cancer (Stockholm III): a multicentre, randomised, non-blinded, phase 3,

32. Bujko K, Nowacki MP, Nasierowska-Guttmejer A, Michalski W, Bebenek M, Kryj M. Long-term result of a randomized trial comparing preoperative shortcourse radiotherapy with preoperative conventionally fractionated chemoradiation for rectal cancer. $\mathrm{B}$ J Surg 2006;93(10):1215-23.

33. Pietrzak L, Bujko K, Nowacki MP, Kepka L, Oledzki J, Rutkowski A, et al; Polish Colorectal Study Group. Quality of life, anorectal and sexual functions after preoperative radiotherapy for rectal cancer: report of a randomised trial. Radiother Oncol 2007;84(3):21725

34. Bujko K, Nowacki MP, Kepka L, Oledzki J, Bebenek M, Kryj M; Polish Colorectal Study Group. Postoperative complications in patients irradiated pre-operatively for rectal cancer: report of a randomised trial comparing short-term radiotherapy vs chemoradiation Colorectal Dis 2005; 7(4):410-6.

35. Ngan SY, Fisher R, Goldstein D, Solomon M, Burmeister B, Ackland SP, et al. A randomized trial comparing local recurrence (LR) rates between short-course (SC) and long-course (LC) preoperative radiotherapy (RT) for clinical T3 rectal cancer: An intergroup trial (TROG, AGITG, CSSANZ, RACS). I Clin Oncol 2010;28(no 15 Suppl):3509.

36. Mohiuddin M, Marks J, Marks G. Management of rectal cancer: short- vs. long-course preoperative radiation. Int J Radiat Oncol Biol Phys 2008;72(3):636-43.

37. Bujko K, Kepka L, Michalski W, Nowacki MP. Doe rectal cancer shrinkage induced by preoperativ radio(chemo)therapy increase the likelihood of anterior resection? A systematic review of randomised trials. Radiother Oncol 2006;80(1):4-12.

38. Wong RK, Tandan V, De Silva S, Figueredo A. Preoperative radiotherapy and curative surgery for the management of localized rectal carcinoma. Cochran Database Syst Rev 2007:(2):CD002102.

39. Quirke P, Steele R, Monson J, Grieve R, Khanna S,
Couture I, et al; MRC CR07/NCIC-CTG CO16 Trial Investigators; NCRI Colorectal Cancer Study Group. Effect of the plane of surgery achieved on local recurrence in patients with operable rectal cancer: a prospective study using data from the MRC CR07 and NCIC-CTG CO16 randomised clinical trial. Lancet 2009;373(9666):821-8

40. Bujko K, Wyrwicz L, Rutkowski A, Malinowska M, Pietrzak L, Kryński J, et al. Long-course oxaliplatinbased preoperative chemoradiation versus $5 \times 5$ Gy and consolidation chemotherapy for $\mathrm{cT} 4$ or fixed $\mathrm{cT} 3$ rectal cancer: results of a randomized phase III study. Ann Oncol 2016;27(5):834-42.

41. Nilsson PJ, van Etten B, Hospers GA, Påhlman L, van de Velde CJ, Beets-Tan RG, et al. Short-course radiotherapy followed by neo-adjuvant chemotherapy in locally advanced rectal cancer-the RAPIDO trial. BMC Cancer 2013:13:279.

42. Francois Y, Nemoz CJ, Baulieux J, Vignal J, Grandjean JP, Partensky C, et al. Influence of the interval between preoperative radiation therapy and surgery on downstaging and on the rate of sphincter-sparing surgery for rectal cancer: the Lyon R90-01 randomized trial. Clin Oncol 1999;17(8):2396

43. Nagtegaal ID, Marijnen CA, Kranenbarg EK, Mulder-Stapel A, Hermans J, van de Velde CJ, et al; Pathology Review Committee. Short-term preoperative radiotherapy interferes with the determination of pathological parameters in rectal cancer. J Pathol 2002;197(1):20-7.

44. Marijnen CA, Nagtegaal ID, Klein Kranenbarg E, Hermans J, van de Velde CJ, Leer JW, et al; Pathology Review Committee and the Cooperative Clinical Investigators. No downstaging after short-term preoperative radiotherapy in rectal cancer patients. J Clin Oncol 2001;19(7):1976-84

45. Braendengen M, Tveit KM, Berglund A, Birkemeyer

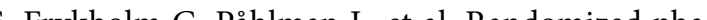
, Frykh study $\mathrm{C}$, Pan $\mathrm{L}$, et al. Rand, Bized phase III study comparing prepertive Ratherapy with chemoradiotherapy in nonresectable rectal cancer. J

46. Yeo SG, Kim DY, Kim TH, Jung KH, Hong YS, Chang $\mathrm{HJ}$, et al. Tumor volume reduction rate measured by magnetic resonance volumetry correlated with pathologic tumor response of preoperative chemoradiotherapy for rectal cancer. Int J Radiat Oncol Biol Phys 2010;78(1):164-71.

47. Bosset JF, Collette L, Calais G, Mineur L, Maingon P, Radosevic-Jelic L, et al; EORTC Radiotherapy Group Trial 22921. Chemotherapy with preoperative radiotherapy in rectal cancer. N Engl J Med 2006;355(11):1114-23.
48. Gérard JP, Conroy T, Bonnetain F, Bouché O, Chapet $\mathrm{O}$, Closon-Dejardin MT, et al. Preoperative radiotherapy with or without concurrent fluorouracil and leucovorin in T3-4 rectal cancers: results of FFCD 9203. Clin Oncol 2006;24(28):4620-5.

49. Gastrointestinal Tumor Study Group. Adjuvant therapy of colon cancer-results of a prospectively randomized trial. N Engl J Med 1984;310(12):737-43.

50. Wagman R, Minsky BD, Cohen AM, Guillem JG, Paty PP. Sphincter preservation in rectal cancer with preoperative radiation therapy and coloanal anastomosis: long term follow-up. Int J Radiat Oncol Biol Phys 1998;42(1):51-7.

51. Grann A, Minsky BD, Cohen AM, Saltz L, Guillem JG, Paty PB, et al. Preliminary results of preoperative 5-fluorouracil, low-dose leucovorin, and concurren radiation therapy for clinically resectable $\mathrm{T} 3$ recta cancer. Dis Colon Rectum 1997;40(5):515-22.

52. Kuvshinoff B, Maghfoor I, Miedema B, Bryer M, Westgate $S$, Wilkes J, et al. Distal margin requirements after preoperative chemoradiotherapy for distal rectal carcinomas: are $<$ or $=1 \mathrm{~cm}$ distal margins sufficient? Ann Surg Oncol 2001;8(2):163-9.

53. Valentini V, Coco C, Cellini N, Picciocchi A, Genovesi D, Mantini G, et al. Preoperative chemoradiation for extraperitoneal T3 rectal cancer: acute toxicity, tumo response, and sphincter preservation. Int J Radiat Oncol Biol Phys 1998;40(5):1067-75.

54. Gerard JP, Chapet O, Nemoz C, Hartweig J, Romestaing P, Coquard R, et al. Improved sphincter preservation in low rectal cancer with high-dose preoperative radiotherapy: the lyon R96-02 randomized trial. J Clin Oncol 2004;22(12):2404-9.

55. Hatfield P, Hingorani M, Radhakrishna G, Cooper R, Melcher A, Crellin A, et al. Short-course radiotherapy, with elective delay prior to surgery, in patients with unresectable rectal cancer who have poor performance status or significant co-morbidity. Rediother Oncol 2009;92(2):210-4.

56. Radu C, Berglund A, Påhlman L, Glimelius B. Shortcourse preoperative radiotherapy with delayed surgery in rectal cancer - a retrospective study. Radiother Oncol 2008;87(3):343-9.

57. Probst CP, Becerra AZ, Aquina CT, Tejani MA, Wexner SD, Garcia-Aguilar J, et al; Consortium for Optimizin the Surgical Treatment of Rectal Cancer (OSTRiCh). Extended Intervals after Neoadjuvant Therapy in Locally Advanced Rectal Cancer: The Key to Improved Tumor Response and Potential Organ Preservation. Am Coll Surg 2015;221(2):430-40.

58. Sun Z, Adam MA, Kim J, Shenoi M, Migaly J, Mantyh CR. Optimal Timing to Surgery after Neoadju- 
vant Chemoradiotherapy for Locally Advanced Rectal Cancer. J Am Coll Surg 2016;222(4):367-74.

59. Huntington CR, Boselli D, Symanowski J, Hill JS, Crimaldi A, Salo JC. Optimal Timing of Surgical Resection After Radiation in Locally Advanced Rectal Adenocarcinoma: An Analysis of the National Cancer Database. Ann Surg Oncol 2016;23(3):877-87.

60. Lefevre JH, Mineur L, Kotti S, Rullier E, Rouanet P, de Chaisemartin C, et al. Effect of Interval (7 or 11 weeks) Between Neoadjuvant Radiochemotherapy and Surgery on Complete Pathologic Response in Rectal Cancer: A Multicenter, Randomized, Controlled Trial (GRECCAR-6). J Clin Oncol 2016;34(31):3773-80.

61. Schrag D, Weiser MR, Goodman KA, Gonen M, Hollywood E, Cercek A, et al. Neoadjuvant chemotherapy without routine use of radiation therapy for patients with locally advanced rectal cancer: a pilot trial. J Clin Oncol 2014;32(6):513-8.

62. Chua YJ, Barbachano Y, Cunningham D, Oates JR, Brown G, Wotherspoon A, et al. Neoadjuvant capecitabine and oxaliplatin before chemoradiotherapy and total mesorectal excision in MRI-defined poor-risk rectal cancer: a phase 2 trial. Lancet Oncol 2010;11(3):241-8.

63. Fernández-Martos C, Pericay C, Aparicio J, Salud A, Safont M, Massuti B, et al. Phase II, randomized study of concomitant chemoradiotherapy followed by surgery and adjuvant capecitabine plus oxaliplatin (CAPOX) compared with induction CAPOX followed by concomitant chemoradiotherapy and surgery in magnetic resonance imaging-defined, locally advanced rectal cancer: Grupo cancer de recto 3 study. J Clin Oncol 2010;28(5):859-65.

64. Fernandez-Martos C, Garcia-Albeniz X, Pericay C, Maurel J, Aparicio J, Montagut C, et al. Chemoradiation, surgery and adjuvant chemotherapy versus induction chemotherapy followed by chemoradiation and surgery: long-term results of the Spanish GCR-3 phase II randomized trial $\dagger$. Ann Oncol 2015;26(8):1722-8.

65. Cercek A, Goodman KA, Hajj C, Weisberger E, Segal $\mathrm{NH}$, Reidy-Lagunes DL, et al. Neoadjuvant chemotherapy first, followed by chemoradiation and then surgery, in the management of locally advanced rectal cancer. J Natl Compr Canc Netw 2014;12(4):513-9.

66. Garcia-Aguilar J, Chow OS, Smith DD, Marcet JE, Cataldo PA, Varma MG, et al; Timing of Rectal Cancer Response to Chemoradiation Consortium. Effect of adding mFOLFOX6 after neoadjuvant chemoradiation in locally advanced rectal cancer: a multicentre, phase 2 trial. Lancet Oncol 2015;16(8):957-66.

67. George TJ, Yothers G, Hong TS, Russel MM, You YN, Parker W, et al. NRG-GI002: A phase II clinical trial platform for total neoadjuvant therapy (TNT) in rectal cancer. J Clin Onc 2018;36(no 4 suppl).

68. Habr-Gama A, Perez RO, Proscurshim I, Campos FG, Nadalin W, Kiss D, et al. Patterns of failure and survival for nonoperative treatment of stage $\mathrm{c} 0$ distal rectal cancer following neoadjuvant chemoradiation therapy. J Gastrointest Surg 2006;10(10):1319-28.

69. Habr-Gama A, Gama-Rodrigues J, Perez RO. Is tailoring treatment of rectal cancer the only true benefit of long-course neoadjuvant chemoradiation? Dis Colon Rectum 2013;56(2):264-6.

70. Habr-Gama A, Sabbaga J, Gama-Rodrigues J, São Julião GP, Proscurshim I, Bailão Aguilar P, et al. Watch and wait approach following extended neoadjuvant chemoradiation for distal rectal cancer: are we getting closer to anal cancer management? Dis Colon Rectum 2013;56(10):1109-17.

71. Maas M, Beets-Tan RG, Lambregts DM, Lammering G, Nelemans PJ, Engelen SM, et al. Wait-and-see policy for clinical complete responders after chemoradiation for rectal cancer. J Clin Oncol 2011;29(35):4633-40.

72. Martens MH, Maas M, Heijnen LA, Lambregts DM, Leijtens JW, Stassen LP, et al. Long-term Outcome of an Organ Preservation Program After Neoadjuvant Treatment for Rectal Cancer. J Natl Cancer Inst 2016;108(12). pii: djw171.

73. Trial Evaluating 3-year Disease Free Survival in Patients With Locally Advanced Rectal Cancer Treated With Chemoradiation Plus Induction or Consolidation Chemotherapy and Total Mesorectal Excision or Nonoperative Management. Secondary Trial Evaluating 3-year Disease Free Survival in Patients With Locally Advanced Rectal Cancer Treated With Chemoradiation Plus Induction or Consolidation Chemotherapy and Total Mesorectal Excision or Non-operative Management. Available at: https://clinicaltrials.gov/ct2/ show/NCT02008656. Accessed May 29, 2018.

74. Observation Versus Surgical Resection in Patients With Rectal Cancer Who Achieved Complete Clinical Response After Neoadjuvant Chemoradiotherapy. Secondary Observation Versus Surgical Resection in Patients With Rectal Cancer Who Achieved Complete Clinical Response After Neoadjuvant Chemoradiotherapy. Avilable at: https://clinicaltrials.gov/ct2/ show/NCT02052921. Accessed May 29, 218. 